\title{
Buoyant Plume through a Permeable Porous Layer Located above a Line Heat Source in an Infinite Fluid Space*
}

\author{
Takashi MASUOKA**, Yasuyuki TAKATSU**, Shuji KAWAMOTO**, \\ Hidekazu KOSHINO** and Takaharu TSURUTA**
}

\begin{abstract}
An experimental and analytical study is conducted on the effects of a horizontal porous layer on the development of the buoyant plume arising from a line heat source in an infinite fluid space. Visual observations and the experimental temperature distributions show the expansion and contraction of the plume at the lower and upper interfaces of the permeable layer. Similarity solutions assuming their proper virtual origins can approximately predict the changes of the plume width. Detailed numerical examination indicates that the lateral flow induced near the interfaces causes deviation from the similarity solutions. The vicinity of the interface is understood to be a transition region between the similarity solutions. The numerical analysis adopts the Beavers-Joseph slip boundary condition with the slip coefficient $\alpha$ estimated by $\alpha=$ $1 / \sqrt{\varepsilon}$, where $\varepsilon$ is the porosity of the porous layer. The fairly good agreement between the experimental and numerical results confirms the validity of the slip coefficient.
\end{abstract}

Key Words: Porous Media, Natural Convection, Boundary Condition, Buoyant Plume, Slip Coefficient, Plume Width, Similarity Solution, Virtual Origin

\section{Introduction}

Natural convection in layered porous systems has been studied extensively because of its importance in thermal insulation and geothermal technologies ${ }^{(1)}$. Layering in permeability or thermal conductivity can cause transitions of flow patterns, even with discontinuous changes in wave numbers for layered horizontal systems heated from below ${ }^{(2)-(5)}$. It is also shown that an adjacent fluid space can affect the flow patterns and either enhance or suppress heat transfer characteristics of convection in porous media in an enclosure depending on the fluid and porous parameters ${ }^{(6)-(9)}$. Concerning buoyant convection in an inhomogeneous infinite space, a study of buoyant plumes above concentrated heat sources in layered porous media suggested steep variations in plume width in the vicinity of the layer interfaces ${ }^{(10)}$. However, little is known about the effect of layering on buoyant convection

* Received 16th September, 1993. Paper No. 92-1177

** Department of Mechanical Engineering, Faculty of Engineering, Kyushu Institute of Technology, 1-1 Sensui-cho, Tobata-ku, Kitakyushu 804, Japan above concentrated heat sources in stratified systems consisting of both porous layers and fluid layers. Therefore, it is of interest to determine the proper boundary condition at the interface between the porous and fluid spaces. Beavers and Joseph ${ }^{(11)}$ introduced the concept of the slip boundary condition for the porous interface:

$$
\frac{\partial v_{f}}{\partial X}=\alpha \frac{v_{f}-v_{p}}{\sqrt{k}}
$$

where $v_{f}$ is the fluid velocity along the porous interface of the fluid-layer side, $v_{p}$ is that of the porouslayer side, $k$ the permeability and $\alpha$ the slip coefficient. Many experiments clarified the fundamental nature of the slip boundary condition ${ }^{(12)-(16)}$. Recently, a 'microscopic' numerical analysis ${ }^{(17)}$ was carried out on the slip parameter $\alpha$ of the Beavers-Joseph slip condition. The numerical results suggest that the value of the parameter $\alpha$ is closely approximated by that given by the relation $\alpha=1 / \sqrt{\varepsilon}$ where $\varepsilon$ is the porosity of the porous medium, although the approximate validity of this relation arises from the fact that the fluid and the solid each receive a shear stress from the external flow. Thus in the present study, utilizing this slip parameter $\alpha$, an experimental and theoretical 
examination is performed on the buoyant plume above a concentrated heat source in an inhomogeneous infinite space.

The physical model is shown in Fig. 1, which consists of a line heat source in an infinite fluid space and a horizontal, permeable porous layer. The problem is how the permeable layer modifies buoyant convection above the line heat source in this layered system, to which previous studies assuming similarity solutions for a homogeneous porous medium and a pure fluid system provide a theoretical background.

According to Wooding ${ }^{(18)}$ the centerline temperature $\Delta T_{o p}=\left(T_{o}-T_{\infty}\right)$ and the half-value (halfmaximum) width of the plume $b_{p}$ for the case of a homogeneous porous medium are expressed, respectively, as

$$
\Delta T_{o p}=\left(\frac{3}{4 \sqrt{6}}\right)^{2 / 3}\left(\frac{Q}{\lambda_{p}}\right)\left[\frac{g \beta\left(Q / \lambda_{p}\right) k X}{\nu x_{p}}\right]^{-1 / 3}
$$

and

$$
\frac{b_{p}}{X}=2.16\left(\frac{4 \sqrt{6}}{3}\right)^{1 / 3}\left[\frac{g \beta\left(Q / \lambda_{p}\right) k X}{\nu x_{p}}\right]^{-1 / 3},
$$

where the porous layer is assumed to be homogeneous and isotropic with permeability $k$ and thermal conductivity $\lambda_{p}$. The origin of the coordinate system is fixed at the location of the line heat source. The surrounding fluid temperature $T_{\infty}$, fluid thermal conductivity $\lambda$ and viscosity $\mu$ are assumed to be constant.

For a fluid layer of $\operatorname{Pr}=0.7, \mathrm{Fujii}^{(19)}$ obtained the similarity solutions

$$
\begin{aligned}
& \Delta T_{o f}=0.376\left(\frac{Q}{\lambda_{f}}\right) \frac{1}{P r}\left[\frac{g \beta\left(Q / \lambda_{f}\right)(1 / P r) X^{3}}{\nu^{2}}\right]^{-1 / 5} \\
& \frac{b_{f}}{X}=2.09\left[\frac{g \beta\left(Q / \lambda_{f}\right)(1 / P r) X^{3}}{\nu^{2}}\right]^{-1 / 5}
\end{aligned}
$$

It is experimentally and numerically examined how the above similarity solutions hold for the present

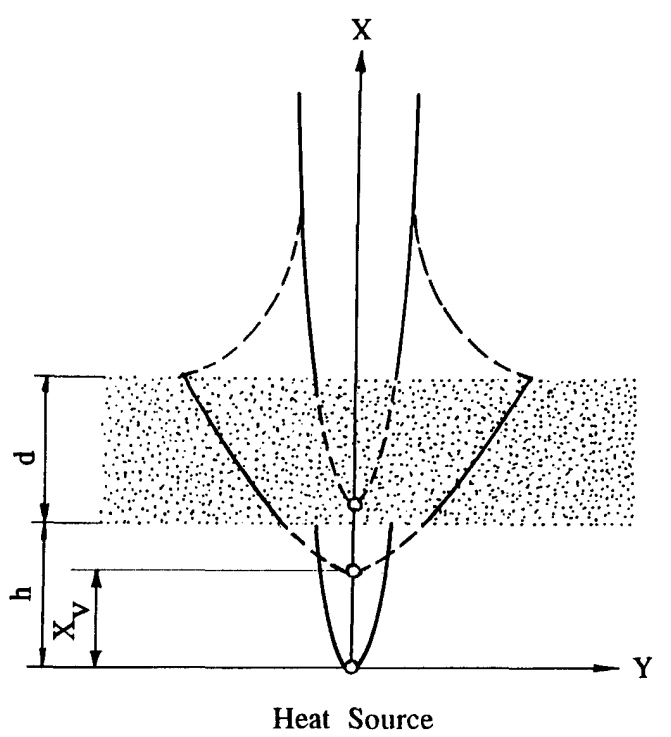

Fig. 1 Physical model and coordinate system layered system, by assuming that the two solutions can be related in such a way that the centerline temperature is continuous at the interface between the porous and fluid spaces. That is, it is thereby considered that the buoyant plumes develop in the porous and fluid spaces from the proper virtual origins, as shown in Fig. 1. Examination of experimental results will be described in the next sections for both the temperature distributions and the variations in the plume width.

\section{Nomenclature}

$B$ : gap of permeable layer

$b:$ half-value (half-maximum) width of buoyant plume

$D a:$ Darcy number $=k / h^{2}$

$d:$ height of permeable layer

$G r:$ Grashof number $=g \beta\left(Q / \lambda_{f}\right) h^{3} / \nu^{2}$

$g:$ gravitational acceleration

$h:$ distance between heat source and lower end of permeable layer

$k:$ permeability

Pr : Prandtl number of fluid

$Q$ : heat release rate from line heat source at unit length

$R a:$ Rayleigh number $=g \beta\left(Q / \lambda_{f}\right) h^{3} / \nu x$

$S_{v}:$ distance from virtual origin $=X-X_{v}$

$T:$ temperature

$v^{*}$ : dimensionless velocity component in $Y^{*}$ direction

$X, Y:$ coordinates defined in Fig. 1

$X_{v}$ : distance between heat source and virtual origin

$X^{*}=X / h$

$Y^{*}=Y / h$

$\alpha$ : Beavers-Joseph slip coefficient

$\beta:$ coefficient of volume expansion

$\varepsilon:$ porosity

$\theta:$ dimensionless temperature $=\left(T-T_{\infty}\right) /\left(Q / \lambda_{f}\right)$

$\lambda$ : thermal conductivity

$\nu:$ kinetic viscosity of fluid

$\varkappa:$ thermal diffusivity

$\Psi:$ stream function

$\Psi^{*}$ : dimensionless stream function

$$
=\Psi_{\nu} /\left\{g \beta\left(Q / \lambda_{f}\right) h^{3}\right\}
$$

Subscripts

$f:$ fluid

$p:$ porous media

$s$ : heat source

$o:$ centerline in plume

$\infty$ : at a distance far apart from plume 


\section{Experimental Apparatus and Procedure}

The experimental apparatus is shown in Fig. 2. An electrically heated platinum wire of $0.5 \mathrm{~mm}$ in diameter with the heating length of $150 \mathrm{~mm}$ is used as the horizontal line heat source. The heat release rate from the heat source is determined from the power input by subtracting the heat conduction loss through the electrodes and the radiation loss. The permeable layer adopted has the horizontal dimensions of 245 $\mathrm{mm} \times 340 \mathrm{~mm}$ and the height $d=10 \mathrm{~mm}$ or $50 \mathrm{~mm}$, which is formed by an array of acrylic resin plates of $2 \mathrm{~mm}$ in thickness with gap spacings $B=2,3$ or $5 \mathrm{~mm}$. The permeable layer is located at the distance $h=20$ $\mathrm{mm}$ above the line heat source. Visual observation of the fluid-region temperature distribution is done using a Mach-Zehnder interferometer together with a $\mathrm{He}^{-}$ $\mathrm{Ne}$ laser beam of the wavelength of $6328 \AA$. The horizontal temperature distributions in the porous array are obtained with a sampling speed of 8 channels per second by shifting 12 calibrated copper-constantan thermocouples of $0.1 \mathrm{~mm}$ in diameter attached to a vertically traversing frame. Measurements are started at about 5 or 6 hours after the initiation of the electrical heating of the line source. All fluid properties are based on the arithmetic mean temperature between the plume center and the surroundings.

Since the flow characteristics through narrow gaps are analogous to those in porous media, the above array of acrylic resin plates is regarded as a porous medium with the equivalent permeability

$$
k=B^{2} \varepsilon / 12 \text {, }
$$

where $B$ is the gap width.

\section{Experimental Results and Discussion}

The experimental temperature distributions at various vertical levels are compared with the similarity solutions in Fig. 3 (a) for the gap width $B=3 \mathrm{~mm}$ and the thickness of the permeable layer $d=10 \mathrm{~mm}$. Figure 3 (b) shows results for $B=3 \mathrm{~mm}$ and $d=50$ $\mathrm{mm}$. In these figures, the symbols $(\bigcirc)$ express the experimental temperature distributions and the solid lines denote the analytical ones with the correspond-

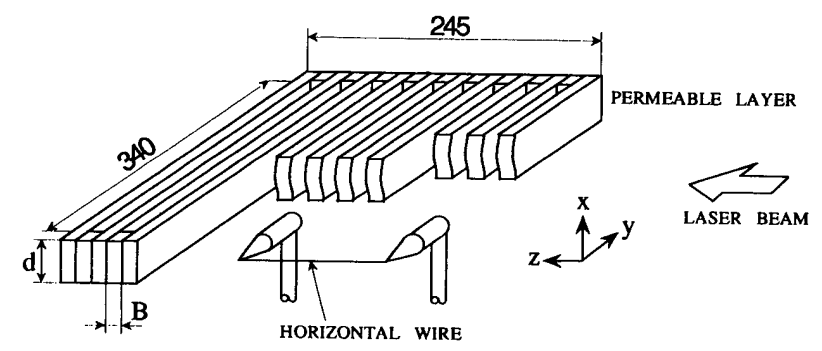

Fig. 2 Experimental apparatus ing plume width. Both experimental and analytical distributions indicate that the plume expands at the lower interface of the permeable layer and contracts at the upper interface, which fundamentally reflects the steep changes in the vertical velocities near the interfaces. Here it is noted that the variations of the plume width are on the same order of magnitude as those predicted by the similarity solutions. Figure 4 shows the typical photographs of the interferential stripes visualizing the isotherms of the plumes, where the plume width increases with decreasing gap width or decreasing permeability. It is seen in Fig. 5 that the tendency of the decrease in the centerline temperature of the plume with increasing permeability of the porous layer can be approximated by the combination of the similarity solutions.

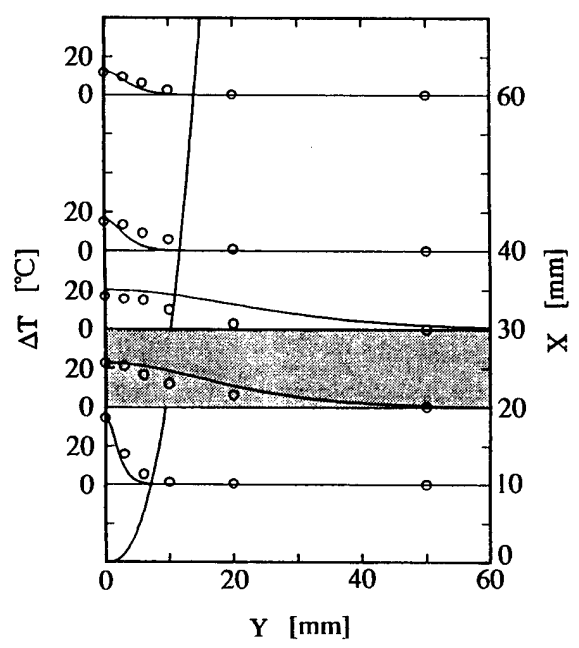

(a)

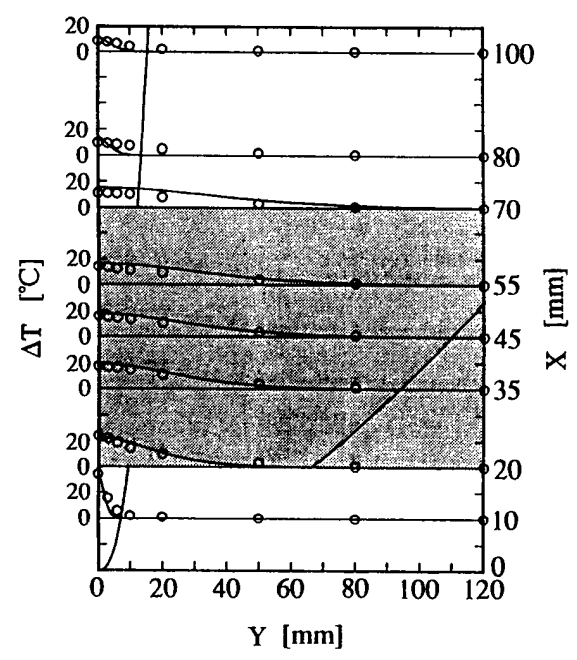

(b)

Fig. 3 Horizontal temperature distributions at $B=3 \mathrm{~mm}$ and $Q=18 \mathrm{~W} / \mathrm{m}$, for ( a ) $d=10 \mathrm{~mm}$ and ( $\mathrm{b}$ ) $d=$ $50 \mathrm{~mm}$ 
The experimental and analytical temperature distributions are considered to show rather good agreement, although a close examination suggests that the experimental temperature distributions not only in the porous region near the lower interface but also in the fluid region near the upper interface deviate more from the similarity solutions at higher tempera-
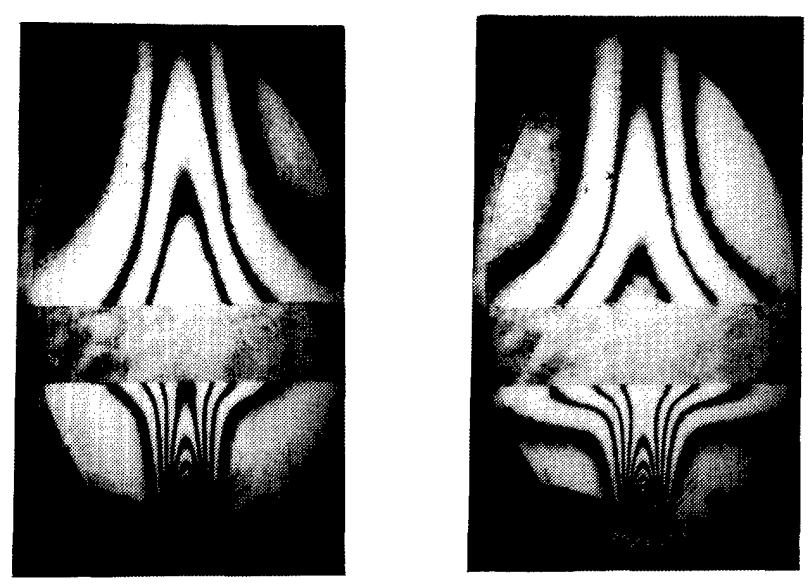

(a) $B=5 \mathrm{~mm}, \mathrm{~d}=10 \mathrm{~mm}$

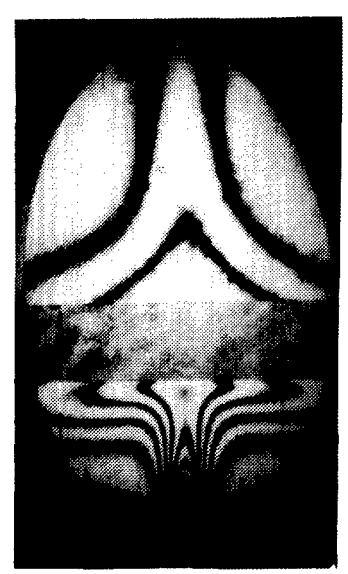

(c) $\mathrm{B}=2 \mathrm{~mm}, \mathrm{~d}=10 \mathrm{~mm}$ (b) $\mathrm{B}=3 \mathrm{~mm}, \mathrm{~d}=10 \mathrm{~mm}$

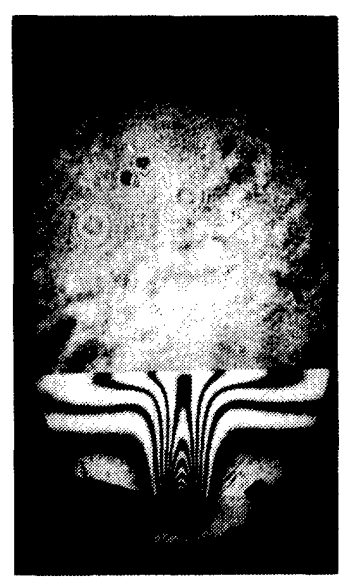

(d) $B=3 \mathrm{~mm}, \mathrm{~d}=50 \mathrm{~mm}$

Fig. 4 Flow visualization of buoyant plume for $Q=18$ $\mathrm{W} / \mathrm{m}$

tures, while the deviations decrease with the increase in height. Thus it can be said that experimentally, the similarity solutions for both fluid and permeable spaces, if related by the continuity of the centerline temperature, can fairly well describe the development of the buoyant plume except in the regions near the interface.

\section{Numerical Analysis}

Two-dimensional, steady numerical calculations are carried out for a two-layer system (Fig. 6) to investigate the effects of a permeable layer on the development of the buoyant plume, where an isothermal plate of finite length is substituted for the line heat source and the heat release rate of the plume from the equivalent line heat source is determined by evaluating the enthalpy transport over a horizontal plane above the isothermal heat source.

The governing equations are written in dimensionless form by introducing stream functions and assuming the Boussinesq approximation.

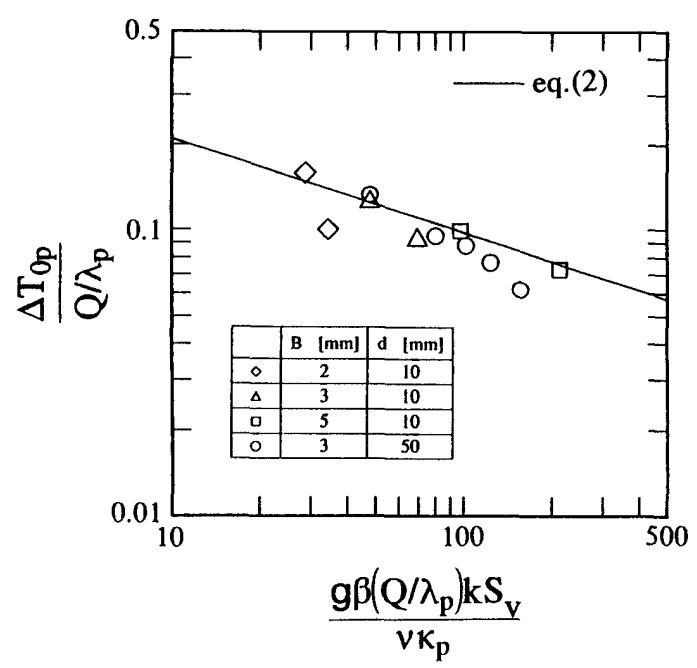

Fig. 5 Variation of centerline temperature with permeability

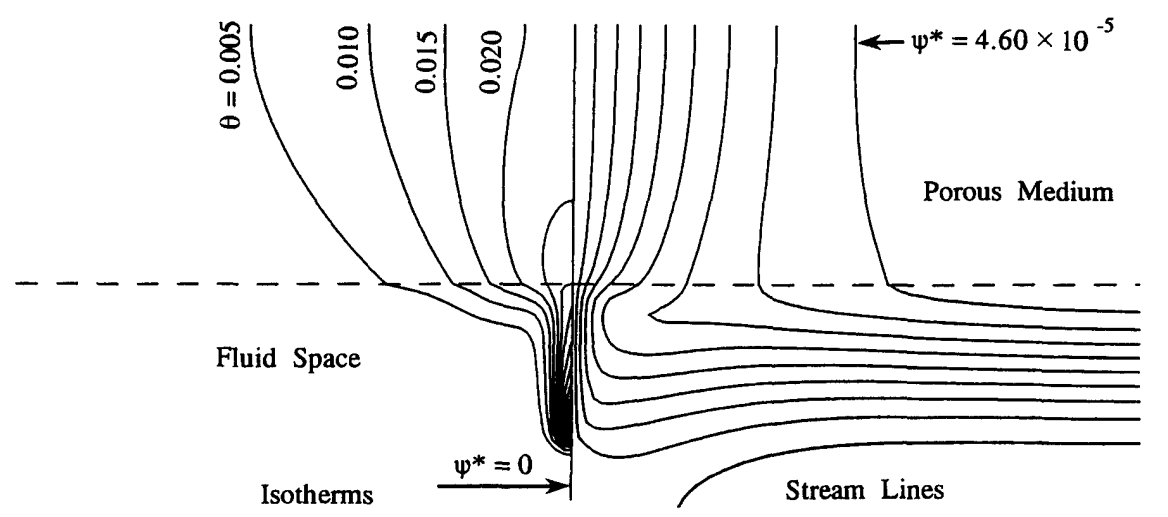

Fig. 6 Numerical flow patterns and isotherms for $R a=7.18 \times 10^{5}, D a=1.88 \times 10^{-3}, \operatorname{Pr}=0.7$ 
Momentum and energy equations for a fluid space are, respectively,

$$
\begin{aligned}
& \frac{\partial \Psi_{f}^{*}}{\partial Y^{*}} \frac{\partial}{\partial X^{*}}\left(\nabla^{2} \Psi_{f}^{*}\right)-\frac{\partial \Psi_{f}^{*}}{\partial X^{*}} \frac{\partial}{\partial Y^{*}}\left(\nabla^{2} \Psi_{f}^{*}\right) \\
& \quad=\frac{1}{G r}\left(\nabla^{4} \Psi_{f}^{*}+\frac{\partial \theta_{f}}{\partial Y^{*}}\right)
\end{aligned}
$$

and

$$
\frac{\partial \Psi_{f}^{*}}{\partial Y^{*}} \frac{\partial \theta_{f}}{\partial X^{*}}-\frac{\partial \Psi_{f}^{*}}{\partial X^{*}} \frac{\partial \theta_{f}}{\partial Y^{*}}=\frac{1}{R a} \nabla^{2} \theta_{f} .
$$

Darcy's law and the energy equation for a porous medium are, respectively,

$$
\frac{\partial^{2} \Psi_{p}^{*}}{\partial X^{* 2}}+\frac{\partial^{2} \Psi_{p}^{*}}{\partial Y^{* 2}}=D a \frac{\partial \theta_{p}}{\partial Y^{*}}
$$

and

$$
\frac{\partial \Psi_{p}^{*}}{\partial Y^{*}} \frac{\partial \theta_{p}}{\partial X^{*}}-\frac{\partial \Psi_{p}^{*}}{\partial X^{*}} \frac{\partial \theta_{p}}{\partial Y^{*}}=\frac{1}{R a} \frac{\chi_{p}}{\chi} \nabla^{2} \theta_{p}
$$

Nondimensional quantities in the above equations are given in the nomenclature.

The calculation is restricted to the right half of the space under the assumption that flow is symmetric with respect to the ordinate. The calculation regime extends from 6 to 7 times the half-width of the plume at the lower interface of the permeable layer. The direction of the inflow at the calculation boundary is assumed to be normal to its boundary surface, where the boundary temperature is specified for the inflow. The outflow is assumed to have fully developed conditions.

Concerning the boundary conditions at the permeable interface between the porous and fluid layers, Masuoka et al. ${ }^{(17)}$ numerically examined the slip parameter $\alpha$, where the flow along the porous medium consisting of parallel flat plates spaced with a narrow gap was simulated from a microscopic point of view, i.e., by applying the Navier-Stokes equation to flow in the narrow gaps instead of the Darcy equation. Their numerical results suggested that the slip parameter $\alpha$ in Eq. (1) is closely approximated by

$$
\alpha=\frac{1}{\sqrt{\varepsilon}}
$$

where $\varepsilon$ is the porosity of the porous medium, and the velocity $v_{p}$ in Eq. (1) is also approximated by the interfacial velocity of the porous-layer side. Then, we may adopt the Beavers-Joseph slip boundary condition combined with Eq. (11).

$$
\frac{\partial v_{f}^{*}}{\partial X^{*}}=-\left(\frac{\alpha}{\sqrt{D a}}\right)\left(v_{f}^{*}-v_{p}^{*}\right)
$$

Also, the following boundary conditions are used at the permeable interface.

$$
\begin{aligned}
& \Psi_{p}^{*}=\Psi_{f}^{*} \\
& \theta_{p}=\theta_{f} \\
& \lambda_{p} \frac{\partial \theta_{p}}{\partial X^{*}}=\lambda_{f} \frac{\partial \theta_{f}}{\partial X^{*}}
\end{aligned}
$$

A numerical analysis is performed using a finitedifference scheme with the numbers of grid points of
70 in the $X^{*}$ direction and 100 in the $Y^{*}$ direction. The value of the Beavers-Joseph slip coefficient $\alpha$ in this calculation is 1.5 .

\section{Numerical Results and Discussion}

The heat source adopted in the numerical analysis has a finite dimension. Little effect of the size of the heat source having a height of the order of the grid size and a thickness of zero is observed, where the average numerical temperature of the heat source is found to be almost the same as the analytical temperature averaged over the height of the heat source using the similarity solution.

Figure 6 shows numerical flow patterns and isotherms when $B=3 \mathrm{~mm}$ and $Q=21.8 \mathrm{~W} / \mathrm{m}$, confirming the similar behaviors observed in Fig. $4(\mathrm{~d})$. The numerical temperature distributions for this case are shown in Fig. 7. These horizontal temperature distributions in the fluid region are almost the same as those of the similarity solutions at slightly above the heat source, although the centerline temperature is somewhat low due to the effect of the heat source of finite length. As the interface is approached, the deviation from the similarity solutions is amplified. It is seen that the numerical plume width becomes larger than that predicted by the similarity solutions near the interface of the permeable layer. This will reflect the deviation of the plume from the boundary layer flow. That is, lateral flow in a fluid space near the interface induces a significant lateral flow in the permeable layer, as shown in Fig. 8, where the velocity slip at the interface is due to the Beavers-Joseph boundary

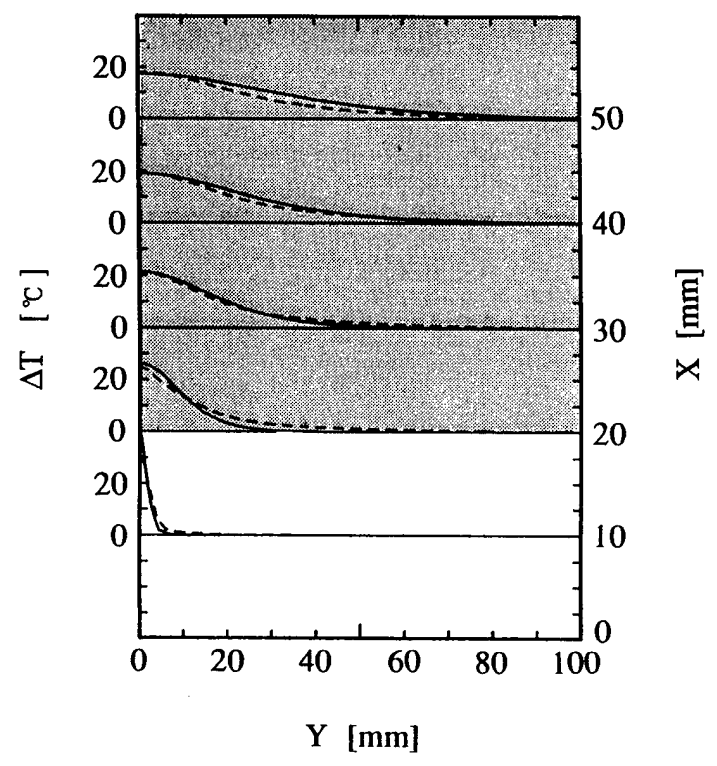

Fig. 7 Comparison between numerical and analytical temperature distributions for $R a=7.18 \times 10^{5}, D a=$ $1.88 \times 10^{-3}, \operatorname{Pr}=0.7$

$(\cdots \cdots$ : numerical, - : analytical $)$ 


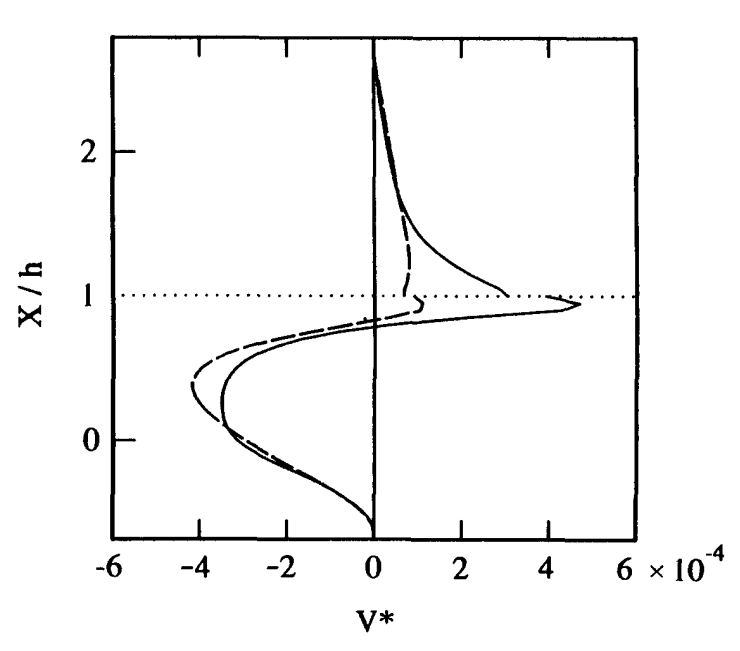

Fig. 8 Numerical lateral velocity distributions for $R a=$ $7.18 \times 10^{5}, D a=1.88 \times 10^{-3}, \operatorname{Pr}=0.7$

$\left.(\cdots \cdots): Y / b_{p X^{*}=1}=1, \longrightarrow: Y / b_{p X^{*}=1}=0.5\right)$

condition. However, this surface effect will not occur deep in the permeable layer. Then, it is natural to expect that the deviation from the boundary layer flow is soon reduced as the height is increased. In other words, the vicinity of the interface is regarded as a transition zone between the two similarity solutions. Taking into account both these numerical results and the visual observations in Fig. 4, the thickness of this zone in the fluid region is estimated to be on the order of the plume width. Thus it is regarded that the agreement between the numerical and similarity temperature distributions is fairly good, except in the vicinity of the interface. Also, we note a fairly good agreement between the experimental and numerical results, which confirms the approximate validity of the slip parameter given by Eq. (11).

\section{Conclusions}

An experimental, analytical and numerical study on buoyant plumes in a permeable layer located above a line heat source in an infinite fluid layer has been conducted and the conclusions are as follows.

(1) The similarity solutions for homogeneous porous and fluid spaces, when related in such a way that the centerline temperature may be continuous, can aptly describe the development of the buoyant plume except in the vicinity of the interfaces.

(2) The detailed numerical examination indicates that the lateral flow induced in the buoyant plume in the near-interface regions causes a deviation from the similarity solutions based on the boundary layer assumption. The vicinity of the interface is understood to be a region of transition between the similarity solutions.

(3) The comparison between the experimental and numerical results confirms the validity of the Beavers-Joseph slip boundary condition with the slip coefficient $\alpha$ estimated by Eq. (11).

\section{Acknowledgement}

The authors wish to express their appreciation to Mr. H. Tanigawa for his assistance.

\section{References}

(1) Masuoka, T., Heat Transfer in Porous Media, J. Heat Transf. Soc. of Jpn., (in Japanese), Vol. 28, No. 108 (1979), p. 147.

(2) Masuoka, T., Convective Currents in a Horizontal Layer Divided by a Permeable Wall, Bull. of JSME, Vol. 17, No. 104 (1974), p. 225.

( 3 ) Masuoka, T., Katsuhara, T., Nakazono, Y. and Isozaki, S., Onset of Convection and Flow Patterns in a Porous Layer of Two Different Media, Heat Transf. Jpn. Res., Vol. 7, No. 2 (1978), p. 39.

(4) McKibbin, R. and O'Sullivan, M. J., Onset of Convection in a Layered Porous Medium Heated from Below, J. Fluid Mech., Vol. 96, Part 2 (1980), p. 375 .

(5) McKibbin, R. and Tyvand, P. A., Anisotropic Modelling of Thermal Convection in Multilayered Porous Media, J. Fluid Mech., Vol. 118 (1982), p. 315.

(6) Poulikakos, D. and Bejan, A., Natural Convection in Vertically and Horizontally Layered Porous Media Heated from the Side, Int. J. Heat Mass Transf., Vol. 26, No. 12 (1983), p. 1805.

(7) Somerton, C. W. and Catton, I., On the Thermal Instability of Superimposed Porous and Fluid Layers, Trans. ASME, J. Heat Transf., Vol. 104 (1982), p. 160.

(8) Bejan, A. and Anderson, R., Natural Convection at the Interface between a Vertical Porous Layer and an Open Space, Trans. ASME, J. Heat Transf., Vol. 105 (1 983), p. 124.

(9) Nishimura, T., Takumi, T., Shiraishi, M., Kawamura, y. and Ozoe, H., Numerical Analysis of Natural Convection in a Rectangular Enclosure Horizontally Divided into Fluid and Porous Regions, Int. J. Heat Mass Transf., Vol. 29, No. 6 (1986), p. 889.

(10) Masuoka, T., Tohda, Y., Tsuruta, T. and Yasuda, Y., Buoyant Convection from Concentrated Heat Sources in Stratified Porous Media, Natural Convection in Porous Media, ASME HTD, Vol. 56 (1986), p. 69 .

(11) Beavers, G. S. and Joseph, D. D., Boundary Conditions at a Naturally Permeable Wall, J. Fluid Mech., Vol. 30, Part 1 (1967), p. 197.

(12) Beavers, C. S., Sparrow, E. M. and Magnuson, R. A., Experiments on Coupled Parallel Flows in a Channel and a Bounding Porous Medium, Trans. ASME, J. Basic Eng., Vol. 92, Series D, No. 1 (1970), p. 843.

(13) Taylor, G. I., A Model for the Boundary Condition 
of a Porous Material. Part 1, J. Fluid Mech., Vol. 49, Part 2 (1971), p. 319.

(14) Saffman, P. G., On the Boundary Conditions at the Surface of a Porous Medium, Studies in Applied Mathematics, Vol. 50 (1971), p. 93.

(15) Williams, W. O., Constitutive Equations for a Flow of an Incompressible Viscous Fluid through a Porous Medium, Quarterly of Applied Mathematics (1978), p. 255.

(16) Rudraiah, N., Coupled Parallel Flows in a Channel and a Bounding Porous Medium of Finite Thickness, Trans. ASME, J. Fluids Eng., Vol. 107 (1985), p. 322.

(17) Masuoka, T., Takatsu, Y., Nakamura, H. and
Tsuruta, T., Channelling Flow and Heat Transfer Characteristics of Porous Insulating Layer, Trans. Jpn. Soc. Mech. Eng., (in Japanese), Vol. 58, No. 551, B (1992), p. 2227.

(18) Wooding, R. A., Convection in a Saturated Porous Medium at Large Rayleigh Number or Peclet Number, J. Fluid Mech., Vol. 15, Part 4 (1963), p. 527.

(19) Fujii, T., Natural Convection above a Horizontal Line Heat Source and a Point Heat Source Theory of the Steady Laminar Flow, Reports of Res. Inst. of Ind. Sci., Kyushu Univ., (in Japanese), Vol. 33 (1962), p. 1. 